\title{
The Frobenius exchange problem on competitions and in classroom
}

\author{
GÉZA KISS
}

Abstract. Let $a_{1}, \ldots, a_{n}$ be relatively prime positive integers. The still unsolved Frobenius problem asks for the largest integer which cannot be represented as $\sum x_{i} a_{i}$ with non-negative integers $x_{i}$, and also for the number of non-representable positive integers. These and several related questions have been investigated by many prominent mathematicians, including Paul Erdős, and a wide range of partial results were obtained by various interesting methods differing both in character and difficulty. In this paper we give a self-contained introduction to this field through problems and comments suitable also for treatment in a class of talented students.

Key words and phrases: Frobenius problem, diophantine equation, mathematical competitions.

ZDM Subject Classification: F69, F64.

\section{The greatest non-representable number}

In this presentation we show some possible educational aspects of an extensive number-theoretical problem. Though there have been published some 200 papers on this topic, as far as we know, nobody has dealt yet with the possibility of applications at school. We hope to enlighten that problems of this type can be used well in teaching talented students.

We also give a sketchy summary of the whole Frobenius problem in order to systemize the material and the exercises in a unified frame.

The first appearance of the coin exchange problem on mathematical competitions was in the academic year 1982/83. 
Problem 1 (International Mathematical Olympics, Paris [14]). Let $a, b, c$ be pairwise relatively prime positive integers. Show that

$$
2 a b c-a b-b c-c a
$$

is the largest integer, which cannot be written in the form

$$
x b c+y c a+z a b,
$$

where $x, y, z$ are non-negative integers.

Problem 2 (Hajós György Competition for Technical College students, Budapest). Someone suggested to mint 3 forint coins besides the existing ones. The idea was based on the opinion that any integer denomination greater than 7 forints can be payed using only 3 and 5 forint coins without exchange. Is this statement true?

Both exercises are special cases of the number-theoretical Frobenius problem, which can be formulated in general as follows: Let $a_{1}<a_{2}<\cdots<a_{n}$ be positive integers, $\operatorname{gcd}\left(a_{1}, \ldots, a_{n}\right)=1$. Find the greatest positive integer $K$, for which the equation $K=\sum_{i=1}^{n} x_{i} a_{i}$ has no solution in non-negative integers $x_{i}$.

We denote this largest positive integer by $G\left(a_{1}, \ldots, a_{n}\right)$, and the number of all positive integers $K$, for which the equation $K=\sum_{i=1}^{n} x_{i} a_{i}$ has no solution (with $x_{i} \geq 0$ ) by $N\left(a_{1}, \ldots, a_{n}\right)$.

Before turning to further problems, let us solve Problem 2. By our new notation we have to prove $G(3,5)=7$. Obviously, the multiples of 3 can be represented. The same is true from 5 for those numbers which give 2 as a residue divided by 3 . The minimal representable number with residue 1 divided by 3 is 10 . Thus in the last two residue classes mod 3 the greatest non-representable elements are 7 and 2, resp. The largest one among them is 7 .

Analogously to the previous proof we can show:

Problem 3. $G\left(a_{1}, a_{2}\right)=\left(a_{1}-1\right)\left(a_{2}-1\right)-1$, if $\operatorname{gcd}\left(a_{1}, a_{2}\right)=1$.

For the proof we have to use, that if $\operatorname{gcd}\left(a_{1}, a_{2}\right)=1$ and $a_{1}<a_{2}$, then $0, a_{2}$, $2 a_{2}, \ldots,\left(a_{1}-1\right) a_{2}$ contain exactly one element from each residue class mod $a_{1}$. We call such numbers a complete residue system mod $a_{1}$.

It is not evident that there always exists a maximal non-representable number also in the case of more than two $a_{i}$ 's. 
Problem 4. If $\operatorname{gcd}\left(a_{1}, \ldots, a_{n}\right)=1$, then there always exists a number $G\left(a_{1}, \ldots, a_{n}\right)$ such that, for $K>G\left(a_{1}, \ldots, a_{n}\right)$ the Diophantine equation $K=$ $\sum_{i=1}^{n} x_{i} a_{i}$ can be solved in non-negative integers.

This exercise, which could be called the fundamental theorem of the Frobenius problem, appeared in the Mathematical and Physical Journal for Secondary Schools in 1997 attached to Béla Vízvári's [18] articles.

We prove the statement by mathematical induction on $n$. The case $n=2$ was indicated in the previous problems. If $n>2$, then assume that the statement is true for $k$ and let us examine it for $k+1$. In the case $n=k+1$ let $d$ be the greatest common divisor of the first $k$ numbers: $d=\operatorname{gcd}\left(a_{1}, \ldots, a_{k}\right)$. By the induction hypothesis, every sufficiently large positive integer can be represented by the numbers $\frac{a_{1}}{d}, \frac{a_{2}}{d}, \ldots, \frac{a_{k}}{d}$. Hence there exists a number $u$ such that $\left(u, a_{k+1}\right)=1$ and $u=v_{1} \frac{a_{1}}{d}+v_{2} \frac{a_{2}}{d}+\ldots+v_{k} \frac{a_{k}}{d}$, where $v_{1}, v_{2}, \ldots, v_{k}$ are non-negative integers. Since $d$ and $a_{k+1}$ are relatively prime, otherwise $a_{1}, a_{2}, \ldots, a_{k+1}$ would have a common divisor greater than 1 , thus also $u d$ and $a_{k+1}$ are relatively prime. Using that the statement is true for $n=2$, we have that with finitely many exceptions every positive integer can be written in the form

$$
x_{1} d u+x_{2} a_{k+1}=x_{1} v_{1} a_{1}+x_{1} v_{2} a_{2}+\ldots+x_{1} v_{k} a_{k}+x_{2} a_{k+1},
$$

where $x_{1} v_{1}, x_{1} v_{2}, \ldots, x_{1} v_{k}, x_{2}$ are non-negative integers.

In spite of the fact that this general result is actually known from the first appearance of the problem, the exact determination of $G\left(a_{1}, \ldots, a_{n}\right)$ seems to be a difficult task in most cases. Already in the case of 3 different coins no general formula can be given. Inserting a new denomination into our set of coins either has no effect on the greatest non-representable number (because the new coin is a multiple of a smaller one, or it is greater than the largest number which was not representable by the previous coins), or, on the contrary, it reduces the limit of representation by enriching tremendously the combinatorial choices of the denominations. In this case the structure of the smallest representatives in the residue classes becomes almost confused.

Therefore, most publications deal with some special conditions in order to make this structure more treatable. This yields actually also the possibility of constructing exercises. Let us examine first the case of three consecutive odd numbers.

Problem 5. Determine $G(2 k+1,2 k+3,2 k+5)$. 
The key for the solution is in looking at the residues of the other two numbers modulo $2 k+1$. These will be multiples of 2 and 4 for every linear combination. The numbers $0,2,4, \ldots, 4 k$ form a complete residue system modulo $2 k+1$. To reach the largest residue we have to use the element $2 k+5$ exactly $k$ times (or, if instead we take $2 k+3$ a few times, we definitely go wronger). Also the smaller residues can be represented from at most this many elements, so the maximal nonrepresentable element is $G(2 k+1,2 k+3,2 k+5)=k(2 k+5)-(2 k+1)=2 k^{2}+3 k-1$.

Roberts [15] proved in 1962 by similar methods that

Problem 6. Let $a_{i}$ form an increasing arithmetical progression: $a_{1}=a$, $a_{2}=a+d, \ldots, a_{n}=a+(n-1) d$, where $\operatorname{gcd}(a, d)=1$. Then

$$
G\left(a_{1}, a_{2}, \ldots, a_{n}\right)=\left\lfloor\frac{a-2}{n-1}\right\rfloor a+(a-1) d .
$$

From the 1960's this problem became quite popular among mathematicians and they determined the exact value of $G\left(a_{1}, \ldots, a_{n}\right)$ in many special cases. There were two important centres: one was led by the German Hofmeister, the other was led by the Norwegian Selmer. The paper of Ramírez Alfonsin [13] published in Bonn in 2000 gives an extensive survey of the most important papers in this topic.

Let us finish this section with solving Problem 1, which can show us with its level of difficulty, with its ideas and methods in the solution, and with its harmonic structure (hopefully) quite a lot from the beauties of the Frobenius problem.

We prove first that $T=2 a b c-a b-b c-c a$ is non-representable in the form $x a b+y b c+z c a$, where $x, y$ and $z$ are non-negative integers. If it were representable, i.e.

$$
x b c+y c a+z a b=2 a b c-a b-b c-c a,
$$

then

$$
(x+1) b c+(y+1) c a+(z+1) a b=2 a b c
$$

would hold. Since for example $b c$ and $a$ are relatively prime, $a$ divides $x+1$, so $a \leq x+1$; analogously $b \leq y+1$, and $c \leq z+1$, which imply

$$
2 a b c=(x+1) b c+(y+1) c a+(z+1) a b \geq 3 a b c,
$$

clearly a contradiction. 
Now we show, that to any positive integer $t^{\prime}$ there exist non-negative (moreover positive) integers $x, y$ and $z$, for which

$$
x b c+y c a+z a b=2 a b c+t^{\prime}
$$

is true. This means that every integer $t>2 a b c$ is representable in the form $x b c+y c a+z a b=t$, where $x, y$, and $z$ are positive integers.

We observe that

$$
b c, 2 b c, 3 b c, \ldots,(a-1) b c, a b c
$$

give different residues divided by $a$, so they form a complete residue system mod $a$, hence one of them, say $x_{1} b c$ is in the same residue class as $t$, so

$$
x_{1} b c \equiv t \quad(\bmod a), \quad 1 \leq x_{1} \leq a .
$$

Analogously, we get that there exist integers $y_{1}$ and $z_{1}$ such that

$$
\begin{aligned}
& y_{1} c a \equiv t \quad(\bmod b), \quad 1 \leq y_{1} \leq b, \\
& z_{1} a b \equiv t \quad(\bmod c), \quad 1 \leq z_{1} \leq c .
\end{aligned}
$$

This implies that

$$
\left(x_{1} b c-t\right)+y_{1} c a+z_{1} a b=x_{1} b c+y_{1} c a+z_{1} a b-t
$$

is divisible by $a$, as well as by $b$ and $c$, and since $a, b$ and $c$ are pairwise relatively prime, it is divisible by $a b c$, so

$$
s=x_{1} b c+y_{1} c a+z_{1} a b \equiv t \quad(\bmod a b c) .
$$

This means that $s$ and $t$, and hence $s-1$ and $t-1$ give the same residue mod $a b c$, namely

$$
\begin{gathered}
t-1=q \cdot a b c+r \\
s-1=q^{\prime} \cdot a b c+r \quad(0 \leq r<a b c) .
\end{gathered}
$$

Here $q \geq 2$, since $t>2 a b c$, and $q^{\prime} \leq 2$, since $s \leq 3 a b c$ by the upper bounds on $x_{1}, y_{1}$ and $z_{1}$. Subtracting the two previous equations we obtain

$$
\begin{gathered}
t-s=\left(q-q^{\prime}\right) a b c \\
t=s+\left(q-q^{\prime}\right) a b c=\left(x_{1}+\left(q-q^{\prime}\right) a\right) b c+y_{1} c a+z_{1} a b,
\end{gathered}
$$


proving the assertion, because $x=x_{1}+\left(q-q^{\prime}\right) a, y=y_{1}$, and $z=z_{1}$ are positive integers.

Let us stay for a few more moments at this problem. We can give a short proof via a more general result which, however, at the same time may hide the essential points of the question.

Johnson [6] published the following statement in 1960, which can be interpreted and verified easily:

Problem 7. Let $a_{1}, a_{2}, a_{3}$ be relatively prime positive integers and $d=$ $\left(a_{1}, a_{2}\right)$ the gcd of the first two numbers. Then

$$
G\left(a_{1}, a_{2}, a_{3}\right)=d \cdot G\left(\frac{a_{1}}{d}, \frac{a_{2}}{d}, a_{3}\right)+(d-1) a_{3} .
$$

At first sight the statement does not seem to be very interesting, but on the one hand it can be largely generalized, and on the other hand it gives a quick solution to Problem 1. We also use that $a \mid a b$ implies $G(a, b, a b)=G(a, b)=$ $a b-a-b$ if $a$ and $b$ are relatively prime. Thus

$G(b c, c a, a b)=c \cdot G(b, a, a b)+(c-1) a b=c(a b-a-b)+(c-1) a b=2 a b c-a b-b c-c a$.

\section{About the number of non-representable integers}

Together with Problem 3 of the previous section also the following related question appeared among the problems of Sylvester [17] in 1884:

Problem 8. We have sufficiently many coins of two different denominations. Determine the number of those positive integers which "cannot be payed" by using these two types of coins without exchange.

Using our earlier notation we want to find the exact value of $N\left(a_{1}, a_{2}\right)$. We make use of the fact that

$$
a_{2}, 2 a_{2}, \ldots,\left(a_{1}-1\right) a_{2}
$$

cover each non-zero residue class $\bmod a_{1}$, i.e. $t a_{2} \equiv k_{t}\left(\bmod a_{1}\right), 1 \leq t \leq a_{1}-1$, where $k_{1}, \ldots, k_{a_{1}-1}$ is a permutation of $1,2, \ldots,\left(a_{1}-1\right)$. These $t a_{2}$ elements will 
be the smallest representable elements of the residue classes. Hence the number of non-representable elements in the $t a_{2}$ residue class is

$$
\left\lfloor\frac{t a_{2}}{a_{1}}\right\rfloor=\frac{t a_{2}-k_{t}}{a_{1}} .
$$

Taking the sum of these numbers over all non-zero residue classes we obtain the total number of the non-representable elements:

$$
\begin{aligned}
\frac{a_{2}-t_{1}}{a_{1}} & +\frac{2 a_{2}-t_{2}}{a_{1}}+\ldots+\frac{\left(a_{1}-1\right) a_{2}-t_{a_{1}-1}}{a_{1}} \\
= & \frac{\left(1+2+\ldots+\left(a_{1}-1\right)\right) a_{2}-\left(t_{1}+t_{2}+\ldots+t_{a_{1}-1}\right)}{a_{1}} \\
= & \frac{\left(1+2+\ldots+\left(a_{1}-1\right)\right)\left(a_{2}-1\right)}{a_{1}}=\frac{\left(a_{1}-1\right)\left(a_{2}-1\right)}{2} .
\end{aligned}
$$

Selmer [16] used the previous method in a more general context. Let $H$ be a complete residue system $\bmod a_{1}$. To every $h \in H$ there exists an $r_{h} \equiv h$ $\left(\bmod a_{1}\right)$, which is representable as $r_{h}=a_{2} y_{2}+a_{3} y_{3}+\ldots+a_{n} y_{n}$ and is the minimal with this property. Then by this notation

$$
N\left(a_{1}, a_{2}, \ldots, a_{n}\right)=\frac{1}{a_{1}} \sum_{h \in H} r_{h}-\frac{a_{1}-1}{2} .
$$

Let us apply this interpretation in further exercises.

Problem 9. Let $a_{i}$ form an arithmetical progression, $a_{1}=a, a_{2}=a+d, \ldots$, $a_{n}=a+(n-1) d$, and write $a-1=p(n-1)+s$, where $0 \leq s<n-1$. Then

$$
N\left(a_{1}, a_{2}, \ldots, a_{n}\right)=\frac{1}{2}((a-1)(p+d)+s(p+1)) .
$$

First we determine the smallest representable $r_{h}$ residues in each class. The numbers $0, d, 2 d, \ldots,(a-1) d$ form a complete residue system $\bmod a$. Our goal is to represent even the largest residue using the least possible number of $a_{i}$ 's. Therefore, we take $a_{n}$ (which has residue $(n-1) d$ ) with the largest possible multiplier $p$ :

$$
p(n-1) \leq a-1<(p+1)(n-1)
$$

This means that

$$
(a-1)=p(n-1)+s ; \quad 0 \leq s<n-1 .
$$


Accordingly, the residue of $p a_{n}+a_{s+1}$ is equal to the residue of $(a-1) d \bmod a$. The $r_{h}$ system can be arranged in a table:

$$
\begin{array}{ccccc}
a_{2} & a_{3} & \ldots & a_{n-1} & a_{n} \\
a_{2}+a_{n} & a_{3}+a_{n} & \ldots & a_{n-1}+a_{n} & 2 a_{n} \\
\ldots & \ldots & \ldots & \ldots & \ldots \\
a_{2}+(p-1) a_{n} & a_{3}+(p-1) a_{n} & \ldots & a_{n-1}+(p-1) a_{n} & p a_{n} \\
a_{2}+p a_{n} & \ldots & a_{s+1}+p a_{n} . & &
\end{array}
$$

The last row occurs only in the case $s>0$.

The sum of the multiples of $d$ in the table is (by the original formula)

$$
d+2 d+\ldots+(a-1) d=d \frac{a(a-1)}{2} .
$$

The sum of the multiples of $a$ is

$$
(n-1) a+2(n-1) a+\ldots+p(n-1) a+(p+1) s a=a\left(\frac{(n-1) p(p+1)}{2}+(p+1) s\right) \text {. }
$$

Hence

$$
\begin{aligned}
N\left(a_{1}, a_{2}, \ldots, a_{n}\right) & =\frac{1}{a} \sum_{h \in H} r_{h}-\frac{a-1}{2} \\
& =\frac{(n-1) p(p+1)}{2}+(p+1) s+\frac{(a-1)}{2}(d-1) \\
& =\frac{1}{2}((p+1)(p(n-1)+2 s)+(d-1)(a-1)) \\
& =\frac{1}{2}((a-1)(p+d)+s(p+1)) .
\end{aligned}
$$

We can also see from the table that the largest non-representable number in the case of $s>0$ is

$$
a_{s+1}+p a_{n}-a=a+s d+p a+p(n-1) d-a=p a+(a-1) d .
$$

If $s=0$, then for the largest non-representable number we get the following formula:

$$
p a_{n}-a=p a+p(n-1) d-a=(p-1) a+(a-1) d .
$$

These two formulas can be combined into one, since for $s>0$,

$$
\left\lfloor\frac{a-1}{n-1}\right\rfloor=\left\lfloor\frac{a-2}{n-1}\right\rfloor=p, \text { whereas for } s=0,\left\lfloor\frac{a-2}{n-1}=p-1\right\rfloor .
$$

Herewith we have proved also the statement of Problem 6 . 
Our next, less known result is related to Problem 1.

Problem 10. Let $a, b, c$ be pairwise relatively prime positive integers. Then the number of positive integers not representable in the form $x b c+y c a+z a b$ with $x, y, z$ non-negative integers is

$$
N(b c, c a, a b)=\frac{2 a b c-b c-c a-a b+1}{2} .
$$

Also in this case we can nicely arrange the smallest elements of each residue class mod $a b$, which are representable with the help of $b c$ and $c a$ :

$$
\begin{array}{ccccc}
0 & b c & 2 b c & \ldots & (a-1) b c \\
c a & b c+c a & 2 b c+c a & \ldots & (a-1) b c+c a \\
\vdots & & & & \vdots \\
(b-1) c a & b c+(b-1) c a & 2 b c+(b-1) c a & \ldots & (a-1) b c+(b-1) c a .
\end{array}
$$

The table contains exactly $a b$ elements. Hence it is enough to prove that they are pairwise incongruent mod $a b$. Otherwise

$$
x_{1} b c+y_{1} c a \equiv x_{2} b c+y_{2} c a \quad(\bmod a b) .
$$

This means that $a b$ divides

$$
x_{1} b c+y_{1} c a-x_{2} b c-y_{2} c a=\left(x_{1}-x_{2}\right) b c+\left(y_{1}-y_{2}\right) c a .
$$

We immediately see that $x_{1}-x_{2}$ is divisible by $a$, and $y_{1}-y_{2}$ is divisible by $b$. Since $0 \leq x_{1}, x_{2} \leq a-1$, and $0 \leq y_{1}, y_{2} \leq b-1$, the divisibility can be true only if $x_{1}=x_{2}$ and $y_{1}=y_{2}$, as claimed.

Let us add now the representatives:

$$
\sum_{i=0}^{a-1} \sum_{j=0}^{b-1}(i b c+j c a)=\frac{a(a-1) b^{2} c}{2}+\frac{b(b-1) a^{2} c}{2}=\frac{a b c}{2}[b(a-1)+a(b-1)] .
$$

We apply the Selmer formula:

$N(a b, b c, c a)=\frac{1}{a b} \cdot \frac{a b c}{2}[b(a-1)+a(b-1)]-\frac{a b-1}{2}=\frac{2 a b c-a b-b c-c a+1}{2}$.

During the determination of the non-representable numbers actually we got a third solution to Problem 1 , because the largest $r_{h} \bmod a b$ is clearly $(a-1) b c+$ $(b-1) c a$. Hence

$$
G(a b, b c, c a)=(a-1) b c+(b-1) c a-a b=2 a b c-a b-b c-c a .
$$


Comparing $N\left(a_{1}, a_{2}, \ldots, a_{n}\right)$ with $G\left(a_{1}, a_{2}, \ldots, a_{n}\right)$ we can make interesting observations. Comparing the formulas of Problems 1 and 10, and also of Problems 3 and 8 , we find that

$$
N\left(a_{1}, a_{2}, \ldots, a_{n}\right)=\frac{G\left(a_{1}, a_{2}, \ldots, a_{n}\right)+1}{2} .
$$

We got important borderline cases of a theorem of Nijenhuis and Wilf [12], who noticed the simple fact that for arbitrary positive integers $x$ and $y$ with $x+y=$ $G\left(a_{1}, a_{2}, \ldots, a_{n}\right)$, at most one of them is representable by $a_{1}, a_{2}, \ldots, a_{n}$, hence

$$
N\left(a_{1}, a_{2}, \ldots, a_{n}\right) \geq \frac{G\left(a_{1}, a_{2}, \ldots, a_{n}\right)+1}{2} .
$$

As an obvious upper bound we have $N\left(a_{1}, a_{2}, \ldots, a_{n}\right) \leq G\left(a_{1}, a_{2}, \ldots, a_{n}\right)$. This bound is sharp, since for

$$
a_{1}=k, a_{2}=k+1, a_{3}=k+2, \ldots, a_{k}=2 k-1
$$

we clearly get $N\left(a_{1}, a_{2}, \ldots, a_{n}\right)=G\left(a_{1}, a_{2}, \ldots, a_{n}\right)=k-1$.

\section{The extremal Frobenius problem}

We saw that the task of determining $G\left(a_{1}, a_{2}, \ldots, a_{n}\right)$ seems to be almost impossible in many cases. Perhaps this is the main reason that many (mainly upper) estimates are known, which give upper bounds for the largest non-representable number under various special assumptions. One of the first such results was achieved by Erdős and Graham [3] in 1972 using Kneser's theorem:

$$
G\left(a_{1}, a_{2}, \ldots, a_{n}\right) \leq 2 a_{n-1}\left\lfloor\frac{a_{n}}{n}-a_{n}\right\rfloor .
$$

Probably these estimations gave the inspiration for examining that choosing a fixed number of $a_{i}$ 's below a given bound, in which case is the largest nonrepresentable number maximal. We introduce the following notation:

$$
g(n, t)=\max G\left(a_{1}, a_{2}, \ldots, a_{n}\right)
$$

where each $a_{i}$ is at most $t$ and their gcd is 1 . Also, we shall denote the set $\left\{a_{1}, a_{2}, \ldots, a_{n}\right\}$ by $A$, and the set of integers representable using the elements of $A$ by $S(A)$. 
Of the many nice estimates on $g(n, t)$, I would like to mention here the very strong result by Dixmier [1], which too, uses Kneser's theorem:

$$
g(n, t) \leq 3 v t-v(v+1) n+v^{2}-v-1, \quad \text { where } \quad v=\left\lfloor\frac{t-2}{n-1}\right\rfloor .
$$

Now we determine $g(n, t)$ in some special cases. The solution of the following exercise is straightforward from Problem 3.

Problem 11. Let $t>2$ be positive integer. Then

$$
g(2, t)=(t-1)(t-2)-1
$$

Using Dixmier's previous theorem also the cases $n=3$ and $n=4$ can be settled. Lewin [10] had determined the value of $g(3, t)$ already 20 years earlier:

$$
g(3, t)=\left\lfloor\frac{1}{2}(t-2)^{2}\right\rfloor-1
$$

Approaching the problem from the direction of "too many" coins, Nagata and Matsumura [11] used densitely considerations. Their result can be proved also by induction on $k$ :

Problem 12. Let $n$ and $k$ be positive integers, and $k \leq n-1$. Then

$$
g(n, n+k)=2 k-1 \text {. }
$$

This result was improved by Paul Erdős [2] in a problem proposed by him:

\section{Problem 13.}

$$
\begin{aligned}
g(n, 2 n) & =2 n+1, \\
g(n, 2 n+1) & =\left\{\begin{array}{lll}
2 n+5, & \text { if } n \neq \equiv 2 & (\bmod 3) \\
2 n+3, & \text { if } n \equiv 2 & (\bmod 3)
\end{array} \quad n>2 .\right.
\end{aligned}
$$

The proof of the first, less complicated statement allows us to have an insight also to the methods of proofs of more difficult theorems. First we show, that for each possible $A$ we have

$$
G(A) \leq 2 n+1
$$


We may assume $a_{n}=2 n$, because otherwise

$$
G(A) \leq g(n, 2 n-1)=2(n-1)-1=2 n-3,
$$

by the statement of Problem 12. If $2 n+1$ is the sum of two elements of $A$, then we can insert it into $A$, and use again Problem 12 for the set $A^{\prime}=A \cup\{2 n+1\}$ :

$$
G(A)=G\left(A^{\prime}\right) \leq g(n+1,2 n+1)=2 n-1 .
$$

Hence we can assume that $2 n+1 \neq a_{i}+a_{j}$.

This means that at most one of $L$ and $2 n+1-L$ can be an element of $A$, but since the number of elements in $A$ is $n$, so exactly one of $L$ and $2 n+1-L$ belongs to $A$.

Let us assume first that $a_{n-1}=2 n-1$. Then $2 \notin A$. Let $r>2$ be the smallest element of $A$. So

$$
2 n, 2 n-1, \ldots, 2 n+1-(r-1)=2 n-r+2 \in A .
$$

Adding $r$ to the last two ones

$$
2 n-r+2+r=2 n+2 \in S(A) \text { and } 2 n+1-(r-2)+r=2 n+3 \in S(A) .
$$

Enlarging $A$ by these two numbers we get a set $A^{\prime}$ with $n+2$ elements. Hence

$$
G(A)=G\left(A^{\prime}\right) \leq g(n+2, n+2+n+1)=2(n+1)-1=2 n+1 .
$$

Finally, if $a_{n-1}<2 n-1$, then $2 \in A$ and so each even number is an element of $S(A)$. Adding 2 to the smallest odd number in $A$ (which surely exists, because the elements of $A$ are relatively prime) we can represent every larger odd number too.

Thus we proved that $G(A) \leq 2 n+1$ for every possible set $A$, i.e.

$$
g(n, 2 n) \leq 2 n+1 .
$$

We also have to verify that there exists such an $A$, for which equality holds. Let $A=\{n+1, n+2, \ldots, 2 n\}$. It is clear that $2(n+1)$ and all larger numbers are in $S(A)$, so

$$
G(A)=2 n+1 .
$$

With a significant extension of the previous argument Erdös and Graham [3] proved, that for $k$ fixed, and $n$ sufficiently large $\left(n \geq 9 k^{2}+15 k+2\right)$,

$$
g(n, 2 n+k)=\left\{\begin{array}{lll}
2 n+4 k+1, & \text { if } n-k \neq 1 & (\bmod 3) \\
2 n+4 k-1, & \text { if } n-k \equiv 1 & (\bmod 3)
\end{array}\right.
$$


Their proof is complex, the easier part is the determination of the extremal set $A$ when equality is satisfied.

Problem 14. Let $n$ and $k$ be positive integers $\left(n \geq 9 k^{2}+15 k+2\right)$. Exhibit a set with $n$ relatively prime elements, each less than $2 n+k+1$, and

$$
G(A)=\left\{\begin{array}{lll}
2 n+4 k+1, & \text { if } n-k \neq 1 & (\bmod 3) \\
2 n+4 k-1, & \text { if } n-k \equiv 1 & (\bmod 3)
\end{array}\right.
$$

Consider first the case $n-k \equiv 1(\bmod 3)$. Write $n=3 m+k+1$ and take

$$
A=\bigcup_{i=1}^{2 m+k}\{3 i\} \cup \bigcup_{j=1}^{m+1}\{6 m+3 k+5-3 j\} .
$$

The least element of $S(A)$, which is congruent to 1 modulo 3 is

$$
2(3 m+3 k+2)=6 m+6 k+4,
$$

so

$$
6 m+6 k+1=2 n+4 k-1 \notin S(A) .
$$

Now we turn to the case $n-k \equiv 2(\bmod 3)$. Write $n=3 m+k+2$ and define $A$ as

$$
A=\bigcup_{i=1}^{2 m+k+1}\{3 i\} \cup \bigcup_{j=1}^{m+1}\{6 m+3 k+7-3 j\} .
$$

The least element of $S(A)$, which is congruent to 2 modulo 3 is

$$
2(3 m+3 k+4)=6 m+6 k+8=2 n+4 k+4 .
$$

In the third case we can construct $A$ with similar methods, as well.

Also the determination of $g(n, t)$ is an unsolved problem in many cases. Lev [9] proved that the result (2) of Erdös and Graham can be extended for $t \leq 3 n-2$. The author of the present paper proved in [7], that (1) holds with equality in several further cases not mentioned in Dixmier's paper [1]. Namely, if $2 \leq d<n, 0 \leq k \leq n-d$, and $n-k \equiv 0$ or $-1(\bmod d+1)$, then

$$
g(n, d n+k)=d(d-1) n+2 d k+d^{2}-d-1 .
$$

The constructions giving the largest non-representable number are generated "by two elements" also in these cases. 
Another extremal question of the Frobenius problem is the following: how should we choose $n$ different denominations not larger than $t$ so that the number of non-representable integers should be maximal. The conjecture of Erdoss and Graham [4, p. 86] was that we obtain the most non-representable integers if we choose the $n$ largest consecutive elements. This can be proved by using one of Dixmier's theorems [1, Theorem 2], further, some computation shows that for several values of $n$ and $t$ there exist other extremal sets too, namely the sets used in the proof of (3) produce the same quantity of non-representable integers as the $n$ largest consecutive ones do, though the maximal non-representable integer is far greater in this case [8]. Let us determine this extremal number of nonrepresentable integers:

Problem 15. Let $n$ and $t$ be positive integers, $1<n \leq t$. Write $t=$ $q(n-1)+r$, where $1 \leq r \leq n-1$. Then

$$
N(t-n+1, t-n+2, \ldots, t)=\frac{(t-n+r-1) q}{2} .
$$

Since the numbers $a_{i}=t-n+i$ are consecutive, all integers in the intervals $J_{m}=[m(t-n+1), m t]$ are representable, $m=1,2, \ldots$ Hence the integers without a representation are those situated before $J_{1}$, between $J_{1}$ and $J_{2}, \ldots$, between $J_{m-1}$ and $J_{m}$ as long as these intervals are disjoint, i.e. $(m-1) t<m(t-n+1)$, or equivalently $m(n-1)<t$. Hence the last value is $m=q$. So the number of integers without representation is

$$
\begin{aligned}
& \sum_{m=1}^{q}[m(t-n+1)-(m-1) t-1]=\sum_{m=1}^{q}(t-m n+m-1) \\
& \quad=q t-\frac{q(q+1)}{2}(n-1)-q=\frac{q}{2}[2 t-(q+1)(n-1)-2] \\
& =\frac{q}{2}[t+q(n-1)+r-(q+1)(n-1)-2]=\frac{(t-n+r-1) q}{2} .
\end{aligned}
$$

All the above exercises can be treated in class at a secondary school after a suitable preparation.

Sylvester's article, in which he examined the case of two variables with simple methods, was published in 1884. A whole century passed till this problem appeared on competitions, and also in a problem book for schools [5, Ex. 152 and 158]. We hope that our students who are interested in mathematics can meet with these and similar exercises regularly in the future. 
Acknowledgement. I am grateful to Professor Róbert Freud for his many important suggestions and also for his corrections in the English translation of the manuscript.

\section{References}

[1] J. Dixmier, Proof of a conjecture by Erdős and Graham concerning the problem of Frobenius, J. Number Theory 34 (1990), 198-209.

[2] P. Erdős, Problem P-84, Can. Math. Bull. 14 (1971), 275-277.

[3] P. Erdős and R. L. Graham, On a linear diophantine problem of Frobenius, Acta Arithmetica 21 (1972), 399-408.

[4] P. Erdős and R. L. Graham, Old and New Problems and Results in Combinatorial Number Theory, Monographies de l'Enseignement Mathématique 28, Université de Genève (1980).

[5] T. Fazakas and A. Hraskó (eds), Bergengóc példatár, Matematika feladatok és azok megoldásai 2., (Problem book „Bergengóc 2”), TypoTEX Kiadó, Budapest, 2001.

[6] S. M. Johnson, A linear Diophantine problem, Can. J. Math. 12 (1960), 390-398.

[7] G. Kiss, Extremal Frobenius numbers in some special cases, Annales Univ. Sci. Budapest 44 (2001), 27-31.

[8] G. Kiss, On the extremal Frobenius problem in a new aspect, Annales Univ. Sci. Budapest 45 (2002), 139-142.

[9] V. F. Lev, On the extremal aspect of the Frobenius problem, Journal of Combinatorial Theory, Series A $\mathbf{7 3}$ (1996), 111-119.

[10] M. Lewin, A bound for a solution of a linear Diophantine problem, J. London Math. Soc. 6 (1972), 61-69.

[11] M. Nagata and H. Matsumura, A theorem in elementary arithmetic, Sugaku 13 (1961-62), 161, Math. Rev. 25, (1963), No. 2386 (in Japanese).

[12] M. Nijenhuis and H. S. Wilf, Representation of integers by linear forms in nonnegative integers, J. Number Theory 4 (1972), 98-106.

[13] J. L. Ramirez Alfonsin, The Diophantine Frobenius Problem, Forschungsinstitut für Diskrete Mathematik, Bonn, Report no. 00893, 2000.

[14] I. Reiman, Nemzetközi Matematikai Diákolimpiák 1959-1994, International Olympics in Mathematics for Students 1959-1994, TypoTEX Kiadó, Budapest, 1997, $362-365$.

[15] J. B. Roberts, Note on linear forms, Proc. Amer. Math. Soc. 7 (1956), 465-469.

[16] E. S. Selmer, On the linear diophantine problem of Frobenius, Journal für reine und angewandte Mathematik 293/294 (1) (1977), 1-17.

[17] J. J. Sylvester, Mathematical questions with their solution, Educational Times 41 (1884), 21. 
[18] B. Vízvári, Mit rakjunk a hátizsákba, hogyan váltsunk fel pénzt? I-II, What to put into the knapsack, and how to change money? I-II, Középiskolai Matematikai Lapok, Budapest, 46 (1996), 386-391, 452-457.

GÉZA KISS

BÖRZSÖNY U. 2/C

H-1098 BUDAPEST

HUNGARY

E-mail: kissg@enternet.hu

(Received July, 2003) 